\title{
An improved figure-of-merit equation for op-amp evaluation
}

\author{
Hirokazu Yoshizawa ${ }^{\text {a) }}$ \\ Graduate School of Electronic Engineering, Saitama Institute of Technology, \\ 1690 Fusaiji, Fukaya-shi, Saitama 369-0293, Japan \\ a)yoshiz_h@sit.ac.jp
}

Abstract: An improved figure-of-merit (FoM) equation is proposed for opamp evaluation. This equation has a modification factor to include the effect of the phase margin, which is not considered in a conventional FoM equation. Simulation results confirm that the proposed FoM equation drastically suppresses the effect of load capacitance variations. By using the proposed FoM equation, evaluations of op-amps become more reasonable as compared with the comparisons using the conventional FoM equation.

Keywords: op-amp, figure of merit, gain-bandwidth product, phase margin Classification: Integrated circuits

\section{References}

[1] W. M. C. Sansen: Analog Design Essentials (Springer, 2006) 182.

[2] S. Chatterjee, Y. Tsividis and P. Kinget: IEEE J. Solid-State Circuits 40 (2005) 2373. DOI:10.1109/JSSC.2005.856280

[3] R. S. Assaad and J. Silva-Martinez: IEEE J. Solid-State Circuits 44 (2009) 2535. DOI:10.1109/JSSC.2009.2024819

[4] Z. Yan, P.-I. Mak, M.-K. Law and R. P. Martins: IEEE J. Solid-State Circuits 48 (2013) 527. DOI:10.1109/JSSC.2012.2229070

[5] W. Qu, J.-P. Im, H.-S. Kim and G.-H. Cho: Proc. IEEE ISSCC Dig. Tech. Papers (2014) 290. DOI:10.1109/ISSCC.2014.6757438

[6] Z. Xiao, Z. Qisheng and D. Ming: IEICE Electron. Express 11 (2014) 20131040. DOI:10.1587/elex.11.20131040

[7] Z. Yan, W. Wang, P.-I. Mak, M.-K. Law and R. P. Martins: IEEE Trans. Circuits Syst. II, Exp. Briefs 62 (2015) 246. DOI:10.1109/TCSII.2014.2368972

[8] B. Razavi: Design of Analog CMOS Integrated Circuits (McGraw-Hill, New York, 2001) 354.

\section{Introduction}

When designing an operational amplifier (op-amp) and comparing its performance with those of other op-amps, a figure-of-merit (FoM) equation is typically used [1, $2,3,4,5,6,7]$. This equation is defined such that the FoM is proportional to the gain-bandwidth product (GBW) and the load capacitance, and is reciprocally proportional to the total current [1]. The FoM equation is useful in judging the 
efficiency of op-amp operation in terms of the GBW and the drivability of the load capacitance per unit current.

However, the shortcoming of this FoM equation is that the phase margin of the op-amp is not considered whatsoever. This issue is explained as follows.

First, an op-amp is assumed to consist of two stages (a so-called two-stage opamp). In the two-stage op-amp, a large load capacitance decreases the frequency of the second pole and hence decreases the phase margin. Therefore, the larger the load capacitance, the lower the phase margin. However, the phase margin is not considered in the FoM equation. A large FoM value is no longer meaningful if the phase margin is too small, such that the op-amp oscillates. Even though op-amps can have phase margins larger than $45^{\circ}$, it is difficult to fairly compare several opamps if their phase margins are not equal.

To resolve this shortcoming of the FoM equation, a modified FoM equation, in which the phase margin is considered, is proposed.

\section{Conventional FoM equation}

A conventional FoM equation for GBW is defined as follows:

$$
\mathrm{FoM}=\mathrm{GBW} \times C_{\mathrm{L}} / I_{\text {total }},
$$

where $C_{\mathrm{L}}$ is the load capacitance and $I_{\text {total }}$ is the total current of the op-amp [1].

Since the FoM is proportional to the load capacitance $C_{\mathrm{L}}$, a large load capacitance leads to a large FoM value. However, a large load capacitance decreases the phase margin. The problem is that the phase margin is not incorporated into this FoM equation. Therefore, it is possible to obtain a large FoM value by using a very large load capacitance if the phase margin is sacrificed.

\section{Proposed FoM equation}

The proposed FoM equation is described as follows:

$$
\mathrm{FoM}=\frac{\mathrm{GBW} \times \mathrm{C}_{\mathrm{L}}}{\mathrm{I}_{\text {total }}} \times \mathrm{f}\left(\phi_{\mathrm{M}}\right)
$$

where $\phi_{\mathrm{M}}$ is the phase margin and $f\left(\phi_{\mathrm{M}}\right)$ is a modification factor for considering the effect of the phase margin. The modification factor $f\left(\phi_{\mathrm{M}}\right)$ is defined as follows:

$$
\mathrm{f}\left(\phi_{\mathrm{M}}\right)=\frac{\tan \phi_{\mathrm{M}}}{\tan \phi_{\text {Mref }}},
$$

where $\phi_{\text {Mref }}$ is a phase margin used as a reference value.

In the following, the op-amp is assumed to be a typical two-stage op-amp as shown in Fig. 1. Fig. 2 shows a Bode plot of the two-stage op-amp. Suppose that this op-amp has a unity-gain frequency of $f_{u}$, the second pole $f_{p 2}$, and a phase margin of $\phi_{\mathrm{M} 1}$ with a load capacitance $C_{\mathrm{L} 1}$. Let us assume that $\phi_{\mathrm{M} 1}$ is a reference phase margin $\phi_{\mathrm{Mref}}$. Then the phase margin $\phi_{\mathrm{M} 1}\left(=\phi_{\mathrm{Mref}}\right)$ is calculated as follows:

$$
\phi_{\mathrm{M} 1}=90^{\circ}-\tan ^{-1} \frac{\mathrm{f}_{\mathrm{u}}}{\left|\mathrm{f}_{\mathrm{p} 2}\right|}=\tan ^{-1} \frac{\left|\mathrm{f}_{\mathrm{p} 2}\right|}{\mathrm{f}_{\mathrm{u}}} .
$$

When the load capacitance $C_{\mathrm{L} 1}$ increases to $C_{\mathrm{L} 2}$ (i.e., $C_{\mathrm{L} 2}>C_{\mathrm{L} 1}$ ), the second pole moves from $f_{\mathrm{p} 2}$ to $\mathrm{f}_{\mathrm{p} 2}{ }^{\prime}$. Here $\left|\mathrm{f}_{\mathrm{p} 2}{ }^{\prime}\right|$ is lower than $\left|\mathrm{f}_{\mathrm{p} 2}\right|$. Then the phase margin 


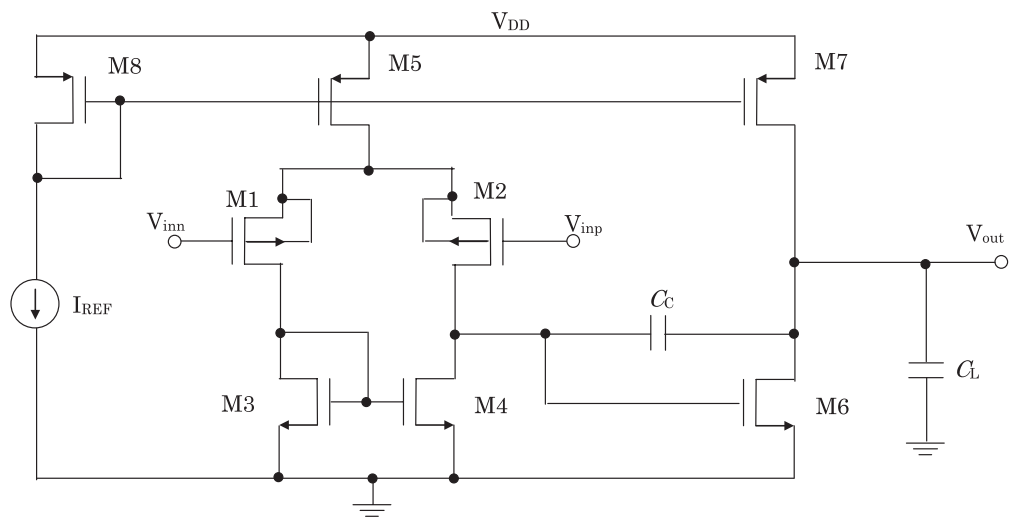

Fig. 1. CMOS two-stage op-amp.

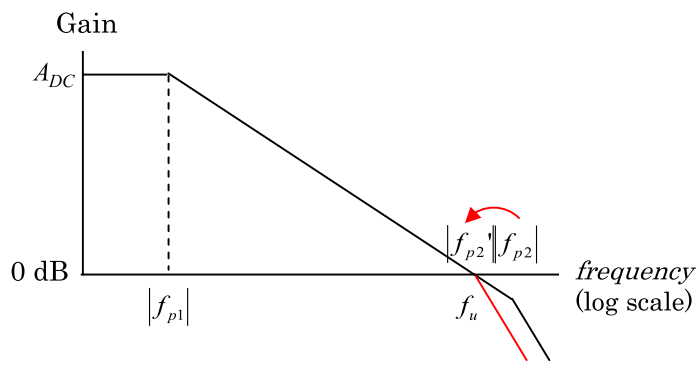

Phase margin $\phi_{\mathrm{M}}$

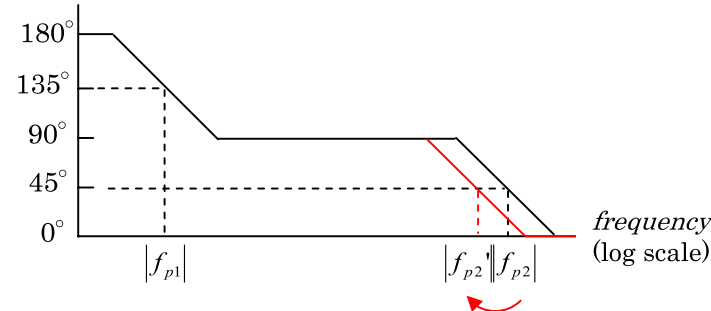

Fig. 2. Bode plot of a two-stage op-amp.

reduces from $\phi_{\mathrm{M} 1}$ to $\phi_{\mathrm{M} 2}\left(\phi_{\mathrm{M} 2}<\phi_{\mathrm{M} 1}\right)$ and the new phase margin $\phi_{\mathrm{M} 2}$ is described as follows:

$$
\phi_{\mathrm{M} 2}=90^{\circ}-\tan ^{-1} \frac{\mathrm{f}_{\mathrm{u}}}{\left|\mathrm{f}_{\mathrm{p} 2^{\prime}}\right|}=\tan ^{-1} \frac{\left|\mathrm{f}_{\mathrm{p} 2}\right|}{\mathrm{f}_{\mathrm{u}}} .
$$

From Eqs. (4) and (5), the following is obtained:

$$
\begin{gathered}
\tan \phi_{\mathrm{M} 1}=\frac{\left|\mathrm{f}_{\mathrm{p} 2}\right|}{\mathrm{f}_{\mathrm{u}}} \text {, and } \\
\tan \phi_{\mathrm{M} 2}=\frac{\left|\mathrm{f}_{\mathrm{p} 2^{\prime}}\right|}{\mathrm{f}_{\mathrm{u}}} . \\
\therefore f\left(\phi_{M 2}\right)=\frac{\tan \phi_{M 2}}{\tan \phi_{M r e f}}=\frac{\tan \phi_{M 2}}{\tan \phi_{M 1}}=\frac{\left|f_{p 2}\right|}{\left|f_{p 2}\right|} \\
=\left(\frac{\mathrm{g}_{\mathrm{m} 6}}{2 \pi \mathrm{C}_{\mathrm{L} 2}}\right) /\left(\frac{\mathrm{g}_{\mathrm{m} 6}}{2 \pi \mathrm{C}_{\mathrm{L} 1}}\right)=\frac{\mathrm{C}_{\mathrm{L} 1}}{\mathrm{C}_{\mathrm{L} 2}},
\end{gathered}
$$

where $g_{\mathrm{m} 6}$ is the transconductance of an NMOS transistor M6 in Fig. 1. 
Then the proposed FoM with $C_{\mathrm{L} 2}$ is as follows:

$$
\begin{gathered}
\text { FoM }\left(\text { with } C_{L 2}\right)=\frac{G B W \cdot C_{L 2}}{I_{\text {total }}} \cdot f\left(\phi_{M 2}\right) \\
=\frac{\mathrm{GBW} \cdot \mathrm{C}_{\mathrm{L} 2}}{\mathrm{I}_{\text {total }}} \cdot \frac{\mathrm{C}_{\mathrm{L} 1}}{\mathrm{C}_{\mathrm{L} 2}}=\frac{\mathrm{GBW} \cdot \mathrm{C}_{\mathrm{L} 1}}{\mathrm{I}_{\text {total }}} .
\end{gathered}
$$

On the other hand, when $C_{\mathrm{L}}=C_{\mathrm{L} 1}$ and the phase margin is $\phi_{M 1}\left(=\phi_{M r e f}\right)$,

$$
\begin{aligned}
& \text { FoM }\left(\text { with } \mathrm{C}_{\mathrm{L} 1}\right)=\frac{\mathrm{GBW} \cdot \mathrm{C}_{\mathrm{L} 1}}{\mathrm{I}_{\text {total }}} \cdot \mathrm{f}\left(\phi_{\mathrm{M} 1}\right) \\
& =\frac{\mathrm{GBW} \cdot \mathrm{C}_{\mathrm{L} 1}}{\mathrm{I}_{\text {total }}} \cdot \frac{\tan \phi_{\mathrm{M} 1}}{\tan \phi_{\mathrm{Mref}}}=\frac{\mathrm{GBW} \cdot \mathrm{C}_{\mathrm{L} 1}}{\mathrm{I}_{\text {total }}} \cdot \frac{\tan \phi_{\mathrm{M} 1}}{\tan \phi_{\mathrm{M} 1}} \\
& =\frac{\mathrm{GBW} \cdot \mathrm{C}_{\mathrm{L} 1}}{\mathrm{I}_{\text {total }}} .
\end{aligned}
$$

$$
\therefore \mathrm{FoM}\left(\text { with } \mathrm{C}_{\mathrm{L} 2}\right)=\mathrm{FoM}\left(\text { with } \mathrm{C}_{\mathrm{L} 1}\right) \text {. }
$$

As shown above, FoM values are equal for the load capacitances of $C_{\mathrm{L} 1}$ and $C_{\mathrm{L} 2}$.

It is well known that a phase margin of $60^{\circ}$ is considered to be the optimum value because of little ringing, fast settling, and robust stability [8]. By setting $\phi_{\text {Mref }}=60^{\circ}$, the general modification factor becomes

$$
\mathrm{f}\left(\phi_{\mathrm{M}}\right)=\frac{\tan \phi_{\mathrm{M}}}{\tan 60^{\circ}} .
$$

Hence, the proposed FoM is as follows:

$$
\mathrm{FoM}=\frac{\mathrm{GBW} \cdot \mathrm{C}_{\mathrm{L}}}{\mathrm{I}_{\text {total }}} \cdot\left(\frac{\tan \phi_{\mathrm{M}}}{\tan 60^{\circ}}\right)
$$

\section{Simulations}

To verify the proposed FoM equation, a two-stage CMOS op-amp was simulated, as shown in Fig. 1, using SPICE parameters for a $0.18-\mu \mathrm{m}$ CMOS process. Table I shows the op-amp device sizes used in HSPICE simulations. The power supply voltage was $1.8 \mathrm{~V}$, and the total current was $178 \mu \mathrm{A}$. With a capacitive load of $10 \mathrm{pF}$, a DC gain of $76 \mathrm{~dB}$, a unity gain frequency of $6.1 \mathrm{MHz}$, and a phase margin of $64^{\circ}$ were obtained.

Fig. 3 shows the load capacitance and phase margin of the op-amp. As expected, the phase margin decreases when the load capacitance increases.

Table I. Op-amp device sizes

\begin{tabular}{|c|c|c|}
\hline device & $\mathrm{W}[\mu \mathrm{m}]$ & $\mathrm{L}[\mu \mathrm{m}]$ \\
\hline $\mathrm{M} 1, \mathrm{M} 2, \mathrm{M} 8$ & 12 & 1.2 \\
\hline $\mathrm{M} 3, \mathrm{M} 4$ & 3.6 & 1.2 \\
\hline $\mathrm{M} 5$ & $12 \times 2$ & 1.2 \\
\hline $\mathrm{M} 6$ & $3.6 \times 8$ & 1.2 \\
\hline $\mathrm{M} 7$ & $12 \times 8$ & 1.2 \\
\hline \multicolumn{3}{|c|}{$I_{\mathrm{REF}}=16 \mu \mathrm{A}, C_{\mathrm{C}}=3 \mathrm{pF}$} \\
\hline
\end{tabular}




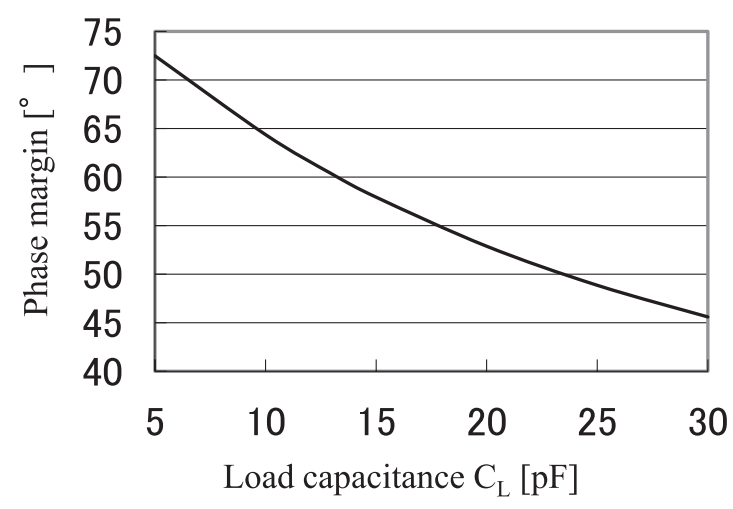

Fig. 3. Phase margin and load capacitance.

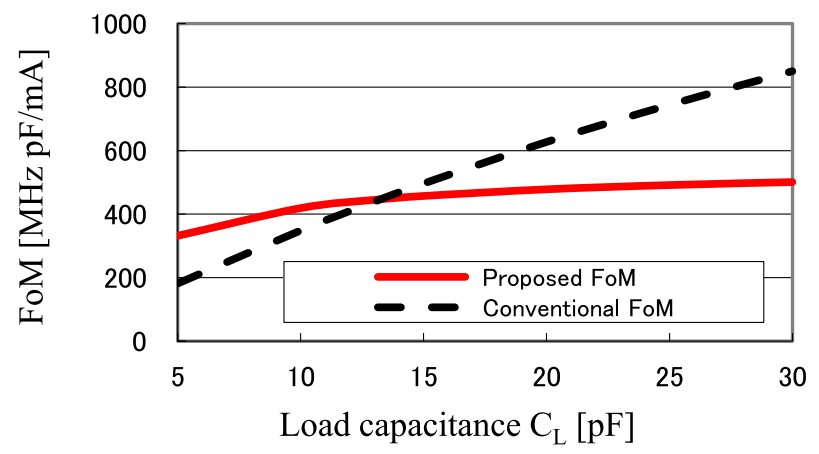

(a)

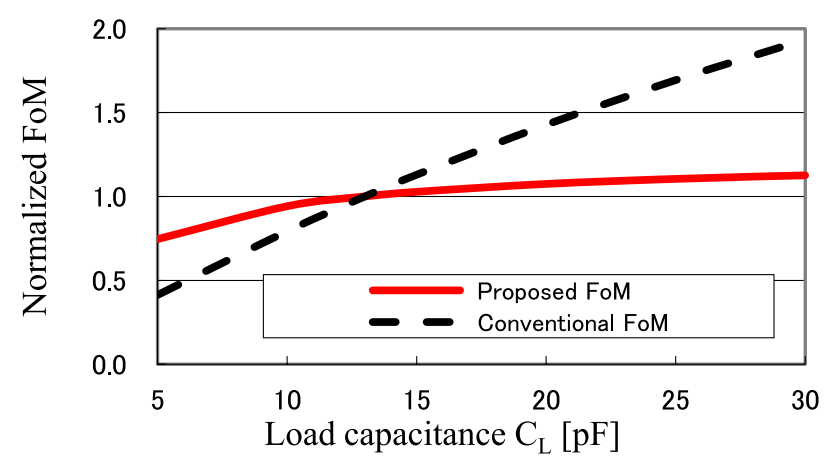

(b)

Fig. 4. FoM and load capacitance. (a) Before normalization. (b) After normalization.

Fig. 4(a) shows load capacitance and FoM values calculated from the simulation results and Eq. (13). As mentioned above, a phase margin of $60^{\circ}$ is considered optimal. Therefore, Fig. 4(a) was normalized using the FoM value at a phase margin of $60^{\circ}$. The normalized FoMs are shown in Fig. 4(b).

Table II shows comparison of the phase margin and FoMs for load capacitances of 5,10 , and $30 \mathrm{pF}$. The proposed FoM equation drastically suppresses the effect of load capacitance variations for a wide range of phase margins. 
Table II. Comparison of the phase margin and FoMs for load capacitances of 5,10 , and $30 \mathrm{pF}$

\begin{tabular}{|c|c|c|c|}
\hline Load capacitance $[\mathrm{pF}]$ & 5 & 10 & 30 \\
\hline Phase margin $\left[^{\circ}\right]$ & 72 & 64 & 45 \\
\hline Conventional FoM $[\mathrm{MHz} \mathrm{pF} / \mathrm{mA}]$ & 181 & 348 & 850 \\
\hline Proposed FoM $[\mathrm{MHz} \mathrm{pF} / \mathrm{mA}]$ & 332 & 419 & 501 \\
\hline Normalized conventional FoM & 0.41 & 0.79 & 1.93 \\
\hline Normalized proposed FoM & 0.75 & 0.94 & 1.13 \\
\hline
\end{tabular}

\section{Conclusion}

In this paper, an improved FoM equation for op-amp evaluation was proposed. This equation includes a modification factor which is related to the phase margin of the op-amp.

Simulation results confirm that the proposed FoM equation drastically suppresses the effect of load capacitance variations, thereby making the comparison and evaluation of op-amps more reasonable, as compared with the comparisons using the conventional FoM equation.

\section{Acknowledgments}

This work is supported by VLSI Design and Education Center (VDEC), the University of Tokyo in collaboration with Synopsys, Inc., and Rohm Corporation. 